Zubarenko O.V. ${ }^{1}$, Koval Larisa ${ }^{1}$, Doykova Katherina ${ }^{2}$, Kopiyka Ganna ${ }^{1}$, Skrypnyk V.V. ${ }^{2}$, Lotysh Nadiya ${ }^{1}$, Papinko R.M. ${ }^{1}$

\title{
Orphan Disease In Structure of Recurrent Lower Respiratory Tract Diseases In Children From One To Five
}

${ }^{1}$ Odessa National Medical University, Odessa, Ukraine

${ }^{2}$ City Children's Clinical Hospital Academician B.J. Reznik, Odessa, Ukraine

\begin{abstract}
The paper contains structure analysis of the lower respiratory tract recurrent disease in 180 children aged one to five years, residents of Odessa, who were treated in pulmonology department of Children's Academician BJ Reznik City Clinical Hospital.

The examinees are represented by two groups: 150 children with recurrent wheezing and 30 - with recurrent pneumonia. The selection criteria were: abnormality of central nervous system, abnormality, cystic fibrosis, congenital heart defects, tuberculosis, human immunodeficiency virus-infection.

The analysis detected that in the survey sample with recurrent lower respiratory tract disease 3.88\% of children had Orphan disease. Thus in the group of children with recurrent wheezing were diagnosed 2 cases of congenital lobar hyperexpansion (ORPHA 1928), one case of lung bronchogenic cyst (ORPHA 2357), one case of pulmonary hypoplasia in the lower lobe of the left lung (ORPHA 2257), and one case of primary ciliary dyskinesia (ORPHA 98,861).

Regarding the entire structure of recurrent wheezing, most frequently was detected atopic asthma (49.33\%). In $24.6 \%$ cases the cause of recurrent wheezing was associated with the otolaryngological pathology. Bronchopulmonary dysplasia was diagnosed in two children.
\end{abstract}

Recurrent pneumonia cases were differentiated

\section{Larisa Koval}

Ukraine, Odessa, Dacha Kovalevsky 81, 65038

email : larikuk@gmail.com

phone: +380974394569 if the localization of process was at the same or different places. When one-sided localization of pneumonia was established, next congenital airway malformations were diagnosed -3 cases of congenital cystic adenomatoid malformations (type I). Genetic pathology often occurred in cases with different lesions location .

In all cases of uncertain diagnosis, predictors of recurrent lower respiratory tract diseases were revealed. Keywords Children, recurrent lower respiratory tract diseases, Orphan diseases.

Keywords: Children, recurrent lower respiratory tract diseases, Orphan diseases.

\section{Introduction}

Respiratory diseases hold first place in the structure of morbidity of children. Over the past decade has been observed an increased incidence of respiratory diseases by $21.5 \%$ [1]. Diseases of the lower respiratory tract are among the most severe diseases of childhood. Special attention should be given to the recurrent course of the disease, which can lead to a chronic process and, finally to child's disability $[2,3,4]$. The causes of recurrent lower respiratory tract diseases may have different pathophysiological mechanisms, but the same type of clinical symptoms often can lead to late diagnosis and delayed treatment $[5,6]$. Nowadays it is relevant to study the rare or the so-called Orphan pathology. Monitoring of rare diseases despite the code of international 
classification of diseases require ORPHA- Results number in additional classification.

\section{Materials and methods}

The aim of the research was to analyze Orphan pathology as a cause of recurrent lower respiratory tract disease among children from one to five nowadays.

A retrospective analysis was performed in two groups, 150 children - with recurrent wheezing (boys $-54.00 \%$, girls $-46.00 \%$ ) and 30 children with recurrent pneumonia (boys - 53.33\%, girls - 46, 66\%), who were hospitalized in the city children's hospital. All children aged from one to five were residents of the city of Odessa (more than 1 million inhabitants is officially registered). Patients were included to the analysis if they experienced two or more radiographically confirmed pneumonias or repeated 3 or more episodes of wheezing. Criteria of exclusion were: abnormality (pathology) of central nervous system, abnormality, cystic fibrosis, congenital heart defects, tuberculosis, human immunodeficiency virus-infection, because this children were sent to specialized centers.

Supervision of patients was conducted in accordance with national standards and international recommendations: included clinical, immunological (concentration of $\operatorname{IgA}$ in serum, $\operatorname{IgM}, \operatorname{IgG}, \operatorname{IgE}$ ), biochemical (level of creatinine, urea, C-reactive protein, Alpha-1 Antitrypsin in serum, sweat chloride), instrumental methods (electrocardiogram, echocardiogram, spirography, chest radiograph (Siemens R50-A; Mobile X-ray Toshiba KCD12MC), computed tomography (Siemens Somatom Sensation, 16 partitions).

Selection and investigation of patients corresponded the principles of biomedical ethics.
Analysis of the causes of recurrent lower respiratory tract disease based on complete physical examination revealed that the most frequently diagnosed atopic asthma $-49.33 \%$ (95\% CI: 41.33 : 57.33 ) (of which $58.10 \%$ were intermittent, and $41.90 \%$ were persistent)was diagnosed in those cases where recurrent wheezing was present. The average age of children with recurrent wheezing was $3.23 \pm 0.77$ years. By the time of verification of the diagnosis were marked up to $2-4(3.8 \pm 1.9)$ episodes of wheezing. The diagnosis was established on the basis of the current criteria and was confirmed by the effectiveness of anti-asthmatic step-therapy.

At $24.00 \%$ (95\% CI: 17.17: 30.83) children recurrent wheezing was associated with acute or exacerbation of chronic otolaryngological diseases (adenoiditis, etmoiditis, etc.).

At $1.33 \%$ patients with recurrent wheezing bronchopulmonary dysplasia were defined, basing on typical anamnesis (prematurity, emergency support due to the severity of state at birth: mechanical ventilation after birth, oxygen therapy).

It is significant that in $3.33 \%$ of recurrent wheezing using radiographic techniques Orphan, a rare pathology was detected. Were diagnosed two cases of congenital lobar hyperexpansion (ORPHA 1928) and one case of bronchogenic cyst (ORPHA 2357). Definitive was that these patients were admitted in serious condition with severe respiratory insufficiency. Wherein bronchospasmolytic therapy showed lack of efficacy.

According to scientific sources lobar hyperexpansion $14-15 \%$ of all congenital lung abnormalities, is more common in boys and is localized mainly in the upper lobe of the left lung $[7,8]$. In our observations suffered boys too, in one case, the process was typically localized in the left upper lobe (Figure 1), the other - in the right lobe. 


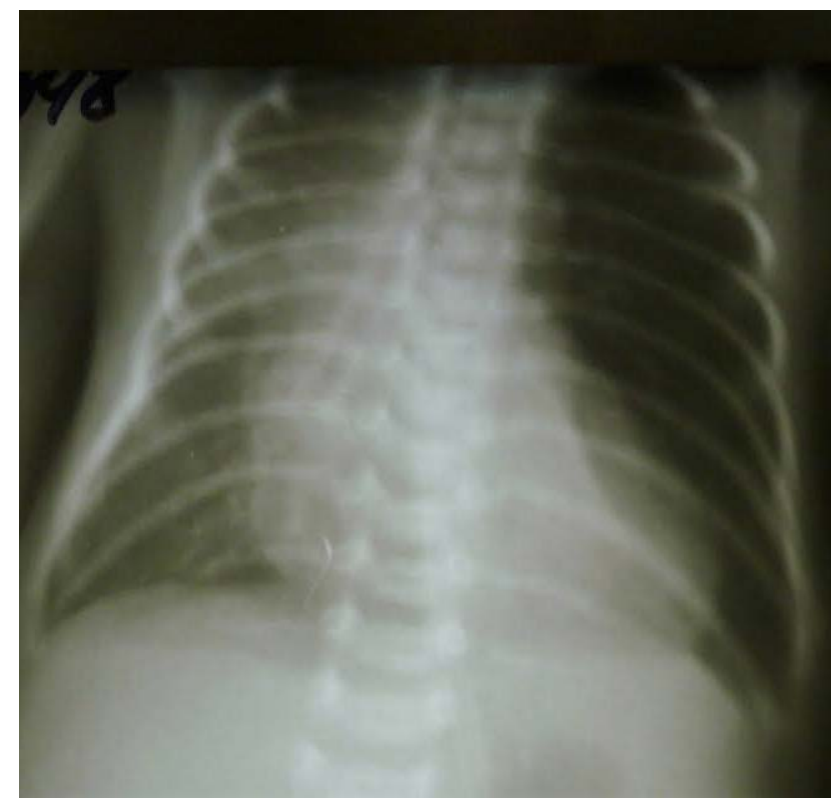

Figure 1 A Boy is 2 years and 6 months old. He is under obser vation from the first days of life in the intensive care unit. The patient was oxygen-dependent from the moment of birth that is why conduction of computed tomography was impossible. The child experienced 4 episodes of wheezing.

Hyperexpansion left upper lobe. Right-sided pneumonia AP x-ray at the age of 42 days: endoprosthesing of endotracheal tube was recommended by the thoracic surgeon in the right intermediate bronchus, located at the Th9 level for selective air administration into the right lung. Mediastinum is displaced to the right, by the enlarged upper lobe of the left lung. The upper lobe of the left lung is evenly hyperlucent, the lower lobe of the left lung is compressed and highlighted by an intense homogeneous region against the background of the mediastinum shadow.

The segmental, median and lower-lobar air bronchi are determined on the right. There is an uneven pneumatization decrease in the projection of the right upper lobe.

In $15-20 \%$ cases bronchogenic cysts are localized in the lung parenchyma and are $20 \%$ cases of all budding bronchial anomaly. The published data indicates that bronchogenic cysts are located in the lower lobes. [8,9]. Absence of lung pattern distinguish bronchogenic cyst from the lobar hyperexpansion. In one child detailed examination revealed cystic hypoplasia type I (ORPHA 2444) in the lower lobe of the left lung.

To Orphan pathology refers Kartagener syndrome (ORPHA 98,861), which is a particular case of primary ciliary dyskinesia, occurs 1: 20000 $[10,8]$. In our study we suppose the Kartagener syndrome in one girl 3.6 years on the basis of identified dextrocardia, recurrent wheezing, chronic rhinosinusitis (Figure 2).

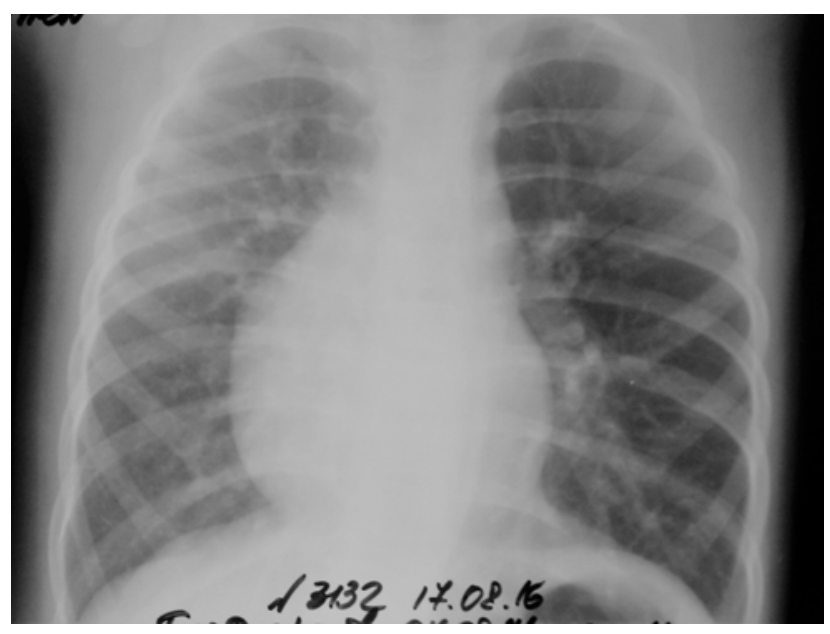

Figure 2 A girl 3 years old. Under the observation from the first year of life.

Dextrocardia. Aneurysm of ventricular septum. Chronic bronchitis. Chronic rhino-sinusitis. One episode of pyelonephritis. Moderate infantile idiopathic scoliosis. Deletion of the short arm of 9 chromosome.

AP x-ray: Dextrocardia, stomach gas bubble is located under the diaphragmatic cupula. The lingular segments in the upper lobe of the left lung are determined by the signs of bronchial dilatation with loss of normal peripheral tapering, and are visualized on the background of deformed lung picture outside exacerbation. S1-3 segments of the left lung are hyperlucent and enlarged. Left cardiodiaphragmatic sinus is smoothed. Parasternal, at the level of lingular segments is defined pleural thickening of the left anterior sinus with obliteration. The left half of the diaphragm is shifted slightly upwards.

In the group of children with recurrent pneumonia the gap between acute episodes ranged from 2 to 9 months (on average $6.77 \pm 3.00$ months). In 3 children the process was localized in the same lobe, and most frequent cystic hypoplasia - type I 
was diagnosed (Figure 3-4). According to the sources congenital cystic adenomatoid malformations (ORPHA 2444) is found in $0.11 \%$ of all newborns $[11,8]$.

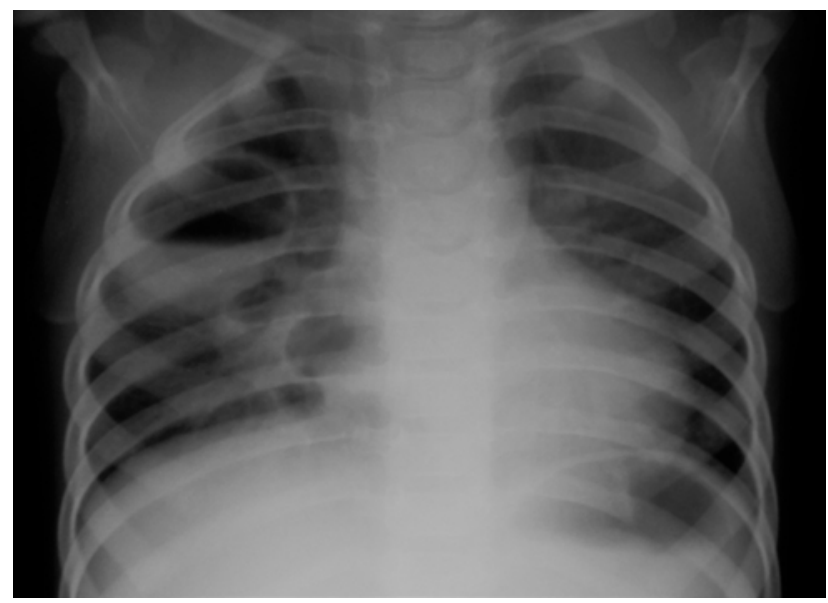

Figure 3 A girl of 2 years old. Under observation from the first days of life in the intensive care unit.

Diagnosis of congenital cystic adenomatoid malformations was established in neonatal period, refered to the subtype I.

AP $x$-ray 1:large cysts $2-5 \mathrm{~cm}$ in diameter with air-fluid levels, surrounded by smaller cysts are detected in the upper and middle lobes of the right lung

In 2 cases, the process was repeated in the additional v.azygos lobe, that was easily detected by the presence of linear shadows of duplicated mediastinal pleura.

In three cases where the recurrent pneumonia had different lesions localization was found genetic pathology. In one girl aged 1 year and 9 months was verified selective IgA deficiency (it was below $0.05 \mathrm{~g} / \mathrm{L}$ repeatedly, concentration of other immunoglobulins remained within the normal range). This pathology occurs with a frequency of 1: 500 to 1: 1000 (ORPHA 69127) [12].

One girl had recurrent pneumonia at the background of Down syndrome. In another case, a child aged 1 year 7 months with a partial deletion of the short arm of 9 chromosome had 3 episodes of pneumonia recorded within one year.

In other cases, a clear nosological entity had not been established, however, all patients had risk factors for recurrent respiratory disease such as low birth weight, undifferentiated connective tissue dysplasia syndrome, asiderotic anemia, etc..

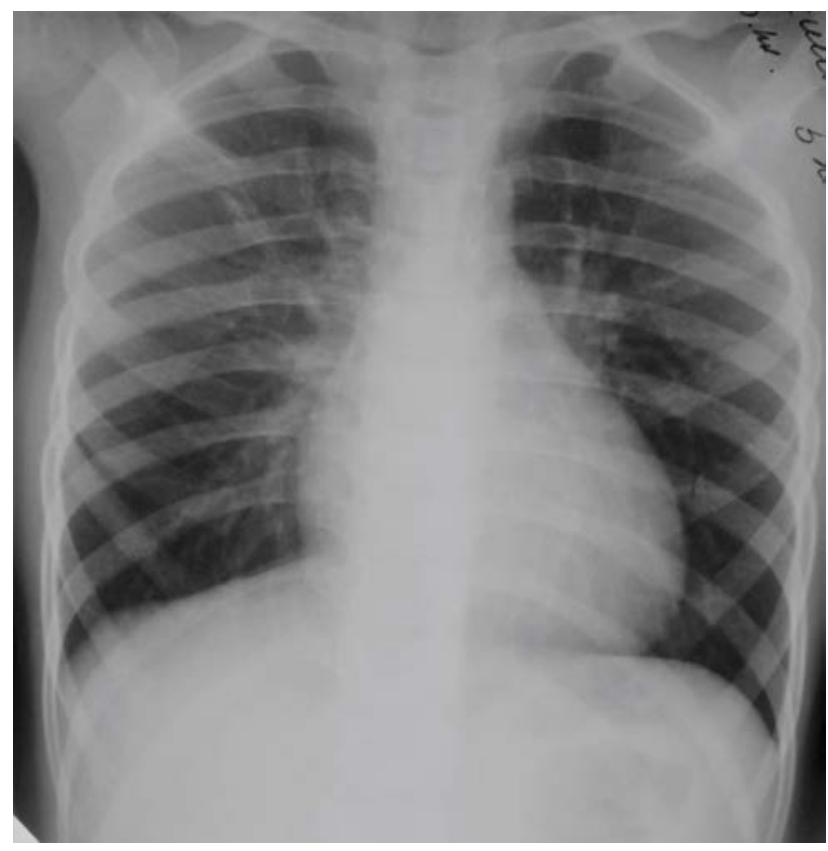

Figure 4 The same girl 5 years old. During 3 years of observation the child experienced several episodes of cysts suppuration, that leaded to the hypoplasia of the lung

AP x-ray 2: fibrous degeneration of lung pattern in the right lung, displacement and deformation of the right diaphragmatic cupula, that indicates diminution of the right lung.

\section{Discussion}

Reports of structure of recurrent lower respiratory disease in children mainly deal with recurrent wheezing $[4,5]$.

Rare to meet is the evidence of recurrent pneumonia. One clear definition of the process has not been accepted by now, making it difficult to diagnose. In pediatric patients there is a suggestion to define recurrent pneumonia as two or more episodes 
of illness per year, or more than three episodes for any period of time, with radiographic evidence clearing the densities between the episodes $[13,14]$. The data on the frequency of Orphan pathology is isolated and fragmentary in the cases of recurrent lower respiratory tract. There is no standardized determination of the prevalence of rare diseases today, in different countries this index ranges from 1: 1000 to 1:200,000. In the European Union the number of patients with Orphan pathology reaches 6-8\%. Information about frequency of localization and gender characteristics differ. [15].

\section{Conclusions}

Our work shows that the recurrent lower respiratory tract disease in children aged one to five, residents of Odessa is variative in its structure. In $3.88 \%$ of cases Orphan pathology was diagnosed. When recurrent wheezing was defined congenital malformation (lobar, bronchogenic cyst), malformations of parenchyma were revealed in cases of recurrent pneumonia with unilateral location, at different locations prevailed genetic pathology.

In all cases of uncertain diagnosis, predictors of recurrent lower respiratory tract diseases were revealed.

\section{References}

1. Antypkin, Y., Chumachenko, N., Lapshin, V. \& Umanets, T. (2016). The aspects of respiratory organs pathological conditions dynamics among child population. Sovremennaya Pediatriya. 2(74), 73-77.

2. Heppe, N., Selivestrova, N., Malishev, V. \& Mashukova, N. (2011). Causes of bronchial obstruction in children and treatment directions. RMJ Mat i ditiya Pediatria. 19(22), 36-39.

3. Zubarenko, A., Portnova, A. \& Stoeva, T. (2007). Basic principles of pathogenesis, diagnosis and differential diagnosis of bronchial obstruction in children. Child's Health.7,14-16.

4. Tenero, L., Piazza, M. \& Piacentini, G. (2016). Recurrent wheezing in children. Translational Pediatrics,5(1), 31-36. http://doi.org/10.3978/j. issn.2224-4336.2015.12.01.

5. Ozdogan, S., Tabakci, B., Demirel, A. S., Atli, B., Besli, G. E. \& Kose, G. (2015). The evaluation of risk factors for recurrent hospitalizations resulting from wheezing attacks in preschool children. Italian Journal of Pediatrics, 41, 91. http://doi.org/10.1186/s13052-015-0201-z.

6. Marini, T., Hobbs, S. K., Chaturvedi, A. \& Kaproth-Joslin, K. (2017). Beyond bronchitis: a review of the congenital and acquired abnormalities of the bronchus. Insights into Imaging, 8(1), 141-153. http://doi.org/10.1007/ s13244-016-0537-y.

7. Kanakis, M., Petsios, K., Bobos, D., Sarafidis, K., Nikopoulos, S., Kyriakoulis, K. \& Giannopoulos, N. (2016). Left Upper Lobectomy for Congenital Lobar Emphysema in a Low Weight Infant. Case Reports in Surgery, 2016, 4182741. http://doi. org/10.1155/2016/4182741.

8. Taussig, L. \& Landau, L. (2008). - Pediatric respiratory medicine (2nd ed.). Philadelphia, USA: Elsevier.

9. Sarper, A., Ayten, A., Golbasi, I., Demircan, A., \& Isin, E. (2003). Bronchogenic Cyst. Texas Heart Institute Journal, 30(2), 105-108.

10. Lobo, J., Zariwala, M. A., \& Noone, P. G. (2015). Primary Ciliary Dyskinesia. Seminars in Respiratory and Critical Care Medicine, 36(2), 169-179. http://doi. org/10.1055/s-0035-1546748.

11. Laudy, J. A. M. \& Wladimiroff, J. W. (2000). The fetal lung 2: pulmonary hypoplasia. Ultrasound Obstet Gynecol, 16: 482-494. doi:10.1046/ j.1469-0705.2000.00252.x.

12. Yel, L. (2010). Selective IgA Deficiency. Journal of Clinical Immunology, 30(1), 10-16. http://doi. org/10.1007/s10875-009-9357-x.

13. Montella, S., Corcione, A. \& Santamaria, 
F. (2017,January) Recurrent Pneumonia in Children: A Reasoned Diagnostic Approach and a Single Centre Experience. Int J Mol Sci.; 18(2). pii: E296. doi: 10.3390/ijms18020296.

14. Patria, F., Longhi, B., Tagliabue, C., Tenconi, R., Ballista, P., Ricciardi, G. \& Esposito, S. (2013). Clinical profile of recurrent community-acquired pneumonia in children. BMC Pulmonary Medicine, 13, 60. http://doi.org/10.1186/14712466-13-60.

15. Kobrinskiy, B., Podpolnaya, M., Bogorad, A., Rozinova, N., Sokolova, L., Gryazina, O. \& Mizernetskiy, Y. (2015). Register of rare chronic lung diseases in children. Vrach i informatsionnie technologie, 3, 64-70. 